\title{
ON THE SMALL AND ESSENTIAL IDEALS IN CERTAIN CLASSES OF RINGS
}

\author{
B. W. GREEN and L. VAN WYK
}

(Received 11 September 1987)

Communicated by B. J. Gardner

\begin{abstract}
It is well known that for a ring with identity the Brown-McCoy radical is the maximal small ideal. However, in certain subrings of complete matrix rings, which we call structural matrix rings, the maximal small and minimal essential ideals coincide.

In this paper we characterize a class of commutative and a class of non-commutative rings for which this coincidence occurs, namely quotients of Prüfer domains and structural matrix rings over Brown-McCoy semisimple rings. A similarity between these two classes is obtained.
\end{abstract} 1980 Mathematics subject classification (Amer. Math. Soc.): 13 F 05, 16 A 42, 16 A 66.

\section{Introduction}

The purpose of this paper is to characterize the essential ideals in certain classes of commutative and non-commutative rings and to characterize those rings in these classes for which the maximal small and minimal essential ideals coincide.

Throughout this paper ring will mean associative ring with identity, and ideal will mean two-sided ideal. Recall that an ideal $A$ in a ring $R$ is

(i) small if $A+B \neq R$ for every proper ideal $B$ in $R$, and, dually,

(ii) essential if $A$ intersects every non-zero ideal in $R$ contrivially.

Loi and Wiegandt [3] showed that the Brown-McCoy radical $\mathscr{G}(R)$ of $R$ is the maximal small ideal. However, if $R$ is not Brown-McCoy semisimple, then $\mathscr{G}(R)$

(C) 1989 Australian Mathematical Society $0263-6115 / 89 \$ A 2.00+0.00$ 
may even be the minimal essential ideal. (As the intersection of two essential ideals is essential, a minimal essential ideal, if such an ideal exists, is unique.)

In Section 2 we give a characterization of the essential ideals in the quotients of a Prüfer domain. Necessary and sufficient conditions are given for the existence of the minimal essential ideal. The characterization of those quotients having the property that the maximal small and minimal essential ideals coincide, shows that such quotients are atypical in the class of rings which are quotients of Prüfer domains.

In Section 3 we contrast the results of Section 2 with those obtained in subrings of complete matrix rings which are rings solely by virtue of the shape (structure) of the matrices. We call them structural matrix rings. It is shown that a structural matrix ring has a minimal essential ideal if and only if the underlying ring has a minimal essential ideal. In that case it coincides with the Brown-McCoy radical if and only if the underlying ring is Brown-McCoy semisimple with no proper essential ideals and a partially ordered set (poset) describing the shape of the matrices has the same property as a certain partially ordered set of ideals in the valuation rings determined by the Prüfer domains in Section 2.

\section{Essential ideals in quotients of Prüfer domains}

Throughout this section $D$ will be a Prüfer domain with quotient field $K$. If $P$ is a prime ideal in $D$ we use $e$ (superscript) to denote the extension (localization) of an ideal in $D$ to the valuation ring $D_{P}$, and $c$ (superscript) to denote the contraction of an ideal in $D_{P}$ to $D$. Also I will be an ideal in $D$ and $\phi: D \rightarrow D / I$ the canonical epimorphism. We denote the set of maximal ideals in $D$ containing $I$ by $\left\{M_{\tau}\right\}_{\tau \in \Upsilon}$. Then (see [2, page 43]) $I=\bigcap_{\tau \in \Upsilon} I_{\tau}$, where $I_{\tau}=I^{e_{\tau} c_{\tau}}$.

It will also be useful to recall some basic properties from the theory of valuations, the details of which can be found in [2] or [4]. Let $V$ be a valuation ring with valuation $v$ and value group $\Gamma$. Then there is a one-to-one correspondence between the ideals $J$ in $V$ and the upper classes of $\Gamma$ given by $J \leftrightarrow v(J)=\{\gamma \in \Gamma: \gamma=v(a)$ for some $a \in J\}$, where an upper class is a set $\Omega$ of positive elements of $\Gamma$ such that $\beta>\alpha$ for $\alpha \in \Omega$ implies that $\beta \in \Omega$. If $\gamma$ is a non-negative element of $\Gamma$, then $\Omega_{\gamma}$ will denote the upper class $\{\beta \in \Gamma: \beta \geq \gamma\}$. Two valuation rings $V_{1}$ and $V_{2}$ having the same quotient field $K$ are said to be independent if 0 is the only common prime ideal. We use $v_{\tau}$ and $\Gamma_{\tau}$ for the valuation and value group of $D_{M_{\tau}}, \tau \in \Upsilon$. For a finite set of pairwise independent valuation rings we shall frequently make use of the approximation theorem for independent valuations (see [2, page 282]). 
If $D$ is a Prüfer domain such that

(i) every non-zero ideal in $D$ is contained in at most finitely many maximal ideals in $D$, and

(ii) for distinct maximal ideals $M_{1}$ and $M_{2}$ in $D$ the valuation rings $D_{M_{1}}$ and $D_{M_{2}}$ are independent, then $D$ will be said to satisfy Condition 1.

THEOREM 2.1. Let $A$ be an ideal in $D$. The ideal $\phi(A)$ is essential in $D / I$ if and only if for some $\Sigma \subseteq \Upsilon$ with $I=\bigcap_{\sigma \in \Sigma} I_{\sigma}, I^{e_{\sigma}} \varsubsetneqq A^{e_{\sigma}}$ for every $\sigma \in \Sigma$.

PROOF. Suppose $\phi(A)$ is essential in $D / I$. As $D_{M_{\tau}}$ is a valuation ring for every $\tau \in \Upsilon$, either $I^{e_{r}} \varsubsetneqq A^{e_{\tau}}$ or $A^{e_{\tau}} \subseteq I^{e_{r}}$. Let $\Phi=\left\{\tau \in \Upsilon: A^{e_{r}} \subseteq I^{e_{r}}\right\}$. Note that $\Phi \neq \Upsilon$, or we would have $A \subseteq \bigcap_{\tau \in \Upsilon} A_{\tau} \subseteq \bigcap_{\tau \in \Upsilon} I_{\tau}=I$, contradicting the essentiality of $\phi(A)$. Set $\Sigma=\Upsilon-\Phi$ and $J=\bigcap_{\sigma \in \Sigma} I_{\sigma}$. If $J=I$, we are finished. Suppose $I \varsubsetneqq J$. Then $J \cap A \subseteq\left(\bigcap_{\sigma \in \Sigma} I_{\sigma}\right) \cap\left(\bigcap_{\tau \in \Upsilon} A_{\tau}\right) \subseteq \bigcap_{\sigma \in \Sigma}\left(I_{\sigma} \cap\right.$ $\left.A_{\sigma}\right) \cap\left(\bigcap_{\tau \in \Upsilon} I_{\tau}\right)=I$. This implies that $\phi(A) \cap \phi(J)=0$ while $\phi(J) \neq 0$, a contradiction.

Conversely, suppose that for some $\Sigma \subseteq \Upsilon$ with $I=\bigcap_{\sigma \in \Sigma} I_{\sigma}, I^{e_{\sigma}} \varsubsetneqq A^{e_{\sigma}}$ for every $\sigma \in \Sigma$. Let $N$ be any non-zero ideal in $D / I$, and set $J=\phi^{-1}(N)$. We show that $\phi(A) \cap N \neq 0$. If $\phi(A) \cap N=0$, then $(A+I) \cap J \subseteq I$, so $A \cap J \subseteq I$. Since $I \varsubsetneqq J$ it follows that $I^{e_{\sigma^{\prime}}} \varsubsetneqq J^{e_{\sigma^{\prime}}}$ for some $\sigma^{\prime} \in \Sigma$. (If $I^{e_{\sigma}}=J^{e_{\sigma}}$ for every $\sigma \in \Sigma$, then $I_{\sigma}=J_{\sigma}$ and $J \subseteq \bigcap_{\sigma \in \Sigma} J_{\sigma}=\bigcap_{\sigma \in \Sigma} I_{\sigma}=I$.) But then $I^{e_{\sigma^{\prime}}} \varsubsetneqq A^{e_{\sigma^{\prime}}} \cap J^{e_{\sigma^{\prime}}}$ as the ideals in $D_{M_{\sigma^{\prime}}}$, form a chain. Furthermore, $A^{e_{\sigma^{\prime}}} \cap J^{e_{\sigma^{\prime}}}=(A \cap J)^{e_{\sigma^{\prime}}}$, and so $I^{e_{\sigma^{\prime}}} \varsubsetneqq I^{e_{\sigma^{\prime}}}$, a contradiction.

THEOREM 2.2. Let $A$ and $I$ be non-zero ideals in a Prüfer domain $D$ satisfying Condition 1. The ideal $\phi(A)$ is essential in $D / I$ if and only if $I^{e_{\tau}} \varsubsetneqq A^{e_{r}}$ for every $\tau \in \Upsilon$.

PROOF. We prove only the necessity, sufficiency being as in Theorem 2.1. Suppose $\phi(A)$ is essential and $A^{e_{r^{\prime}}} \subseteq I^{e_{r^{\prime}}}$ for some $\tau^{\prime} \in \Upsilon$. Applying the approximation theorem for independent valuations $\left(\left\{M_{\tau}\right\}_{\tau \in \Upsilon}\right.$ is finite), we choose $x \in K$ such that

$$
v_{\tau}(x) \in v_{\tau}\left(I^{e_{\tau}}\right) \text { if } \tau \neq \tau^{\prime} \text {, and } \quad v_{\tau^{\prime}}(x) \notin v_{\tau^{\prime}}\left(I^{e_{r^{\prime}}}\right) \text {. }
$$

Let $J=x D \cap D$. Then $J \nsubseteq I$, for if it were, then $J^{e_{r}} \subseteq I^{e_{r}}$ for every $\tau \in \Upsilon$, contradicting the fact that $J^{e_{\tau^{\prime}}}=(x D \cap D)^{e_{r^{\prime}}}=x D_{M_{\tau^{\prime}}} \cap D_{M_{r^{\prime}}} \supsetneqq I^{e_{r^{\prime}}}$. We have

$$
A \cap J \subseteq\left(\bigcap_{\tau \in \Upsilon} A_{\tau}\right) \cap\left(\bigcap_{\tau \in \Upsilon} J_{\tau}\right) \subseteq\left(\bigcap_{\substack{\tau \in \Upsilon \\ \tau \neq \tau^{\prime}}}\left(A_{\tau} \cap J_{\tau}\right)\right) \cap\left(I_{\tau^{\prime}} \cap J_{\tau^{\prime}}\right) \subseteq I,
$$

a contradiction to the essentiality of $\phi(A)$. 
In the theorems above the linearity of inclusion of ideals when passing to the localization is of crucial importance. The most classic examples of Prüfer domains are of course Dedekind domains. If instead of relaxing the conditions for a Dedekind domain (omitting the Noetherian assumption) so that one still has a Prüfer domain, we omit the requirement that the domain be integrally closed, then the characterization given above is no longer true in general, as is illustrated by the example below.

EXAMPLE 2.3. Let $F$ be a field and $R=F\left[\left[t^{2}, t^{3}\right]\right]$, the ring of formal power series in $t^{2}$ and $t^{3}$. This is a 1-dimensional local noetherian ring with maximal ideal $M=t^{2} R+t^{3} R$. Let $I=t^{2} M, A=t^{2} R$ and $J=t^{3} R$. Then $I \varsubsetneqq A$ and $J \nsubseteq I$. However $A \cap J \varsubsetneqq I$, and so $\phi(A)$ is not essential in $R / I, \phi$ being the canonical epimorphism.

We next consider the existence of a minimal essential ideal in the quotient of a Prüfer domain.

THEOREM 2.4. Let $I$ be a non-zero ideal in a Prüfer domain $D$ satisfying Condition 1. The following are equivalent.

(i) $D / I$ has a minimal essential ideal.

(ii) For every $\tau \in \Upsilon$ the upper class $v_{\tau}\left(I^{e_{\tau}}\right)$ has an immediate predecessor.

ProOF. (i) $\rightarrow$ (ii) Let $N$ be the minimal essential ideal in $D / I$, and let $A=$ $\phi^{-1}(N)$. Suppose that for some $\tau^{\prime} \in \Upsilon, v_{\tau^{\prime}}\left(I^{e_{\tau^{\prime}}}\right)$ does not have an immediate predecessor. By Theorem 2.2, $I^{e_{r}} \varsubsetneqq A^{e_{\tau}}$ for every $\tau \in \Upsilon$. For each $\tau \in \Upsilon$, choose $\gamma_{\tau} \in \Gamma_{\tau}$, the value group of $D_{M_{\tau}}$, such that

$$
\gamma_{\tau} \in v_{\tau}\left(A^{e_{r}}\right)-v_{\tau}\left(I^{e_{\tau}}\right) \quad \text { and } \quad v_{\tau^{\prime}}\left(A^{e_{\tau^{\prime}}}\right) \supsetneqq \Omega_{\gamma_{\tau^{\prime}}} \supsetneqq v_{\tau^{\prime}}\left(I^{e_{r^{\prime}}}\right) .
$$

That this is possible follows immediately from the remarks at the beginning of this section. Applying the approximation theorem for independent valuations there exists $x \in K$ such that $v_{\tau}(x)=\gamma_{\tau}, \tau \in \Upsilon$. Then $x \in \bigcap_{\tau \in \Upsilon} A^{e_{\tau}}$. Let $J=x D \cap D$. Then for each $\tau \in \Upsilon, J^{e_{r}}=x D_{M_{\tau}} \cap D_{M_{\tau}}=x D_{M_{\tau}}$ as $x \in D_{M_{r}}$. Furthermore, $v_{\tau}\left(x D_{M_{r}}\right)=\Omega_{\gamma_{r}}$. Hence $I^{e_{r}} \varsubsetneqq J^{e_{\tau}}$, from the fact that $x \in J^{e_{r}}-I^{e_{r}}$ and the linearity of inclusion of ideals in $D_{M_{\tau}}$. Therefore $\phi(J+I)=\phi(J)$ is essential in $D / I$. But $(J+I)^{e_{r}}=J^{e_{r}}+I^{e_{r}} \subseteq A^{e_{r}}$ for every $\tau \in \Upsilon$, and $(J+I)^{e_{r^{\prime}}} \varsubsetneqq A^{e^{r^{\prime}}}$. This contradicts the fact that $\phi(A)$ is the minimal essential ideal in $D / I$.

(ii) $\rightarrow$ (i) Suppose that for every $\tau \in \Upsilon$, the upper class $v_{\tau}\left(I^{e_{\tau}}\right)$ has an immediate predecessor $\gamma_{r}$. Applying the approximation theorem for independent valuations we choose $x \in K$ such that $v_{\tau}(x)=\gamma_{\tau}, \tau \in \Upsilon$. Let $A=x D \cap D$. Then 
$A^{e_{r}}=x D_{M_{\tau}}$ for every $\tau \in \Upsilon$. One easily sees that $\phi(A)$ is the minimal essential ideal in $D / I$.

The next result shows that the minimal essential ideal (if it exists) and the maximal small ideal, that is the Brown-McCoy radical, in $D / I$ coincide if and only if $M_{\tau}^{e_{r}}$ is principal for every $\tau \in \Upsilon$ and every maximal chain in the poset $\left\{\left(M_{\tau}^{e_{\tau}}\right)^{i}: \tau \in \Upsilon, I^{e_{r}} \subseteq M_{\tau}^{e_{\tau}}, i>0\right\}$ has precisely two elements. This assertion follows directly from the following theorem if one notes that $M_{\tau}^{e_{r}},\left(M_{\tau}^{e_{r}}\right)^{2} \nsubseteq M_{\tau^{\prime}}^{e_{\tau^{\prime}}}$ for $\tau \neq \tau^{\prime}$. (Note the similarity between this condition on the poset and the pair condition in Section 3 (the sentence just before Theorem 3.5).)

THEOREM 2.5. Let I be a non-zero ideal in a Prüfer domain $D$ satisfying Condition 1. Then the maximal small and minimal essential ideals coincide in $D / I$ if and only if $M_{\tau}^{e_{\tau}}$ is principal and $I^{e_{r}}=\left(M_{\tau}^{e_{\tau}}\right)^{2}$ for every $\tau \in \Upsilon$.

ProOF. Suppose the maximal small and minimal essential ideals coincide in $D / I$, and call it $N$. Let $A=\phi^{-1}(N)$. Then $A=\bigcap_{\tau \in \Upsilon} M_{\tau}$, and by Theorem $2.2, I^{e_{r}} \subsetneq A^{e_{r}}=M_{\tau}^{e_{\tau}}$ for every $\tau \in \Upsilon$. We shall prove the implication by contradiction.

Suppose first that $M_{\tau^{\prime}}^{e_{\tau^{\prime}}}$ is not principal for some $\tau^{\prime} \in \Upsilon$. Then the first isolated subgroup of the value group of $D_{M_{r}}$ is not discrete, and so by the approximation theorem for independent valuations we may choose $x \in K$ such that

$$
I^{e_{\tau}} \varsubsetneqq x D_{M_{\tau}} \subseteq M_{\tau}^{e_{\tau}} \quad \text { for } \tau \neq \tau^{\prime}, \quad \text { and } \quad I^{e_{\tau^{\prime}}} \varsubsetneqq x D_{M_{\tau^{\prime}}} \varsubsetneqq M_{\tau^{\prime}}^{e_{\tau^{\prime}}}
$$

Let $J=x D \cap D$. Then $J^{e_{r}} \subseteq A^{e_{r}}$ for every $\tau \in \Upsilon$, and $J^{e_{\tau^{\prime}}} \varsubsetneqq A^{e_{r^{\prime}}}$. Hence $J \varsubsetneqq A$. But $\phi(J)$ is essential by Theorem 2.2, contradicting the choice of $N=\phi(A)$.

Secondly, if for some $\tau^{\prime} \in \Upsilon, M_{\tau^{\prime}}^{e_{\gamma^{\prime}}}$ is principal and $I_{\tau^{\prime}}^{e_{\tau^{\prime}}} \neq\left(M_{\tau^{\prime}}^{e_{\gamma^{\prime}}}\right)^{2}$, then the first isolated subgroup of the value group of $D_{M_{\tau^{\prime}}}$ is discrete, and

$$
I^{e_{r^{\prime}}} \varsubsetneqq\left(M_{\tau^{\prime}}^{e_{r^{\prime}}}\right)^{2} \subsetneq M_{\tau^{\prime}}^{e_{r^{\prime}}}
$$

Again we may choose an ideal $J$ in $D$ such that $\phi(J)$ is essential and $\phi(J) \varsubsetneqq \phi(A)$.

Conversely, suppose $M_{\tau}^{e_{\tau}}$ is principal and $I^{e_{\tau}}=\left(M_{\tau}^{e_{\tau}}\right)^{2}$ for every $\tau \in \Upsilon$. Then the first isolated subgroup of the value group of $D_{M_{\tau}}$ is discrete, and so $v_{\tau}\left(I^{e_{\tau}}\right)$ has an immediate predecessor for every $\tau \in \Upsilon$. By Theorem 2.4, $D / I$ has a minimal essential ideal. Clearly this ideal is the maximal small ideal.

Note that, since we deal with commutative rings in this section, the BrownMcCoy radical is just the Jacobson radical.

In the next section we shall see that certain matrix rings over a ring $R$ with $\mathscr{G}(R)=0$ and no proper essential ideals also have the property described in Theorem 2.5. We conclude this section by showing that the factor rings $D / I$, with $D$ satisfying Condition 1, provide examples of such rings. 
PROPOSITION 2.6. Let I be a non-zero ideal in a Prüfer domain $D$ satisfying Condition 1. Then $\mathscr{G}(D / I)=0$ if and only if $D / I$ has no proper essential ideals.

ProOF. It is easy to see that if $R$ is any commutative ring with only finitely many maximal ideals, then $\mathscr{G}(R)=0$ implies that $R$ has no proper essential ideals.

Conversely, suppose $\mathscr{G}(D / I) \neq 0$, that is $I \varsubsetneqq \bigcap_{\tau \in \Upsilon} M_{\tau}$. Then $I^{e_{\tau^{\prime}}} \varsubsetneqq M_{\tau^{\prime}}^{e_{\tau^{\prime}}}$ for some $\tau^{\prime} \in \Upsilon$. We assert that $\phi\left(M_{\tau^{\prime}}\right)$ is essential in $D / I$. This follows directly from Theorem 2.1, with $\Sigma=\Upsilon$, if one notes that $I^{e_{r}} \varsubsetneqq D_{M_{\tau}}=M_{\tau^{\prime}}^{e_{\tau}}$ for $\tau \neq \tau^{\prime}$.

\section{Essential ideals in structural matrix rings}

Throughout this section $R$ will be an associative ring with identity.

Let $B=\left[b_{i j}\right]$ be an $n \times n$ Boolean matrix. For a non-empty subset $S$ of $R$ we set $\mathbf{M}(B, S):=\left\{X=\left[x_{i j}\right] \in \mathbf{M}_{n}(S): b_{i j}=0 \rightarrow x_{i j}=0\right\}$, and we call $\mathbf{M}(B, S)$ the set associated with $B$ and $S$.

Henceforth $B$ will be a reflexive and transitive $n \times n$ Boolean matrix. The subring $\mathbf{M}(B, R)$ of the complex matrix ring $\mathbf{M}_{n}(R)$ is called a structural matrix ring. The matrix $B$ determines and is determined by the binary relation $\leq_{B}$ on $\mathbf{n}:=\{1,2, \ldots, n\}$ defined by

$$
i \leq_{B} j: \leftrightarrow b_{i j}=1 \text {. }
$$

This quasi-order relation gives naturally rise to the equivalence relation $\sim_{B}$ on n defined by

$$
i \sim_{B} j: \leftrightarrow i \leq_{B} j \text { and } j \leq_{B} i
$$

Let $b$ be the number of equivalence classes induced by $\sim_{B}$, and let $z_{1}, z_{2}, \ldots, z_{b}$ denote their representatives. Then $\leq_{B}$ is a partial order relation on $\left\{z_{a}: a \in\right.$ b\}. For all $a, a^{\prime} \in \mathbf{b}$ such that $z_{a} \leq_{B} z_{a^{\prime}}$, we set $\Lambda_{a a^{\prime}}:=\left\{k \in \mathbf{b}: z_{a} \leq_{B} z_{k}\right.$ and $\left.z_{k} \leq_{B} z_{a^{\prime}}\right\}$. The ideals in $\mathbf{M}(B, R)$ are, by [5, Proposition 1.2], the sets $\mathscr{F}_{f}:=\left\{X=\left[x_{i j}\right] \in \mathbf{M}(B, R): x_{i j} \in f\left(\Lambda_{a a^{\prime}}\right)\right.$ if $i \sim_{B} z_{a}, j \sim_{B} z_{a^{\prime}}$ and $\left.z_{a} \leq_{B} z_{a^{\prime}}\right\}$, corresponding to the set-inclusion preserving functions $f:\left\{\Lambda_{a a^{\prime}}: a, a^{\prime} \in \mathbf{b}\right.$ and $\left.z_{a} \leq_{B} z_{a^{\prime}}\right\} \rightarrow\{I: I$ is an ideal in $R\}$, that is the functions $f$ such that $\Lambda_{a a^{\prime}} \subseteq$ $\Lambda_{d d^{\prime}}$ implies $f\left(\Lambda_{a a^{\prime}}\right) \subseteq f\left(\Lambda_{d d^{\prime}}\right)$. We use $f, g$ and $h$ as generic symbols for these set-inclusion preserving functions.

The essential ideals in $\mathbf{M}(B, R)$ can be characterized in terms of those in $R$ and the maximal elements in the partially ordered (with respect to set-inclusion) set $\left\{\Lambda_{a a^{\prime}}: a, a^{\prime} \in \mathbf{b}\right.$ and $\left.z_{a} \leq_{B} z_{a^{\prime}}\right\}$.

THEOREM 3.1. $\mathscr{I}_{f}$ is an essential ideal in $\mathrm{M}(B, R)$ if and only if $f\left(\Lambda_{a a^{\prime}}\right)$ is essential in $R$ for every maximal $\Lambda_{a a^{\prime}}$. 
PROOF. Let $f\left(\Lambda_{a a^{\prime}}\right)$ be essential in $R$ for every maximal $\Lambda_{a a^{\prime}}$, and let $\mathscr{J}_{g}$ be any non-zero ideal in $\mathbf{M}(B, R)$, say $g\left(\Lambda_{d d^{\prime}}\right) \neq 0$. Let $\Lambda_{k k^{\prime}}$ be a maximal element containing $\Lambda_{d d^{\prime}}$. Then $f\left(\Lambda_{k k^{\prime}}\right) \cap g\left(\Lambda_{k k^{\prime}}\right) \neq 0$, and so $\mathscr{J}_{f} \cap \mathscr{J}_{g} \neq 0$.

Conversely, let $\mathscr{F}_{f}$ be essential in $\mathbf{M}(B, R)$, and consider any maximal $\Lambda_{a a^{\prime}}$. Let $A$ be a non-zero ideal in $R$, and let $g$ be the function that maps $\Lambda_{a a^{\prime}}$ onto $A$ and everything else onto 0 . Then $\mathscr{I}_{f} \cap \mathscr{I}_{g} \neq 0$, and so $f\left(\Lambda_{a a^{\prime}}\right) \cap A \neq 0$.

COROLLARY 3.2. $\mathrm{M}(B, R)$ has a minimal essential ideal if and only if $R$ has a minimal essential ideal.

PrOOF. It follows directly from Theorem 3.1 that if $N$ is the minimal essential ideal in $R$, then $\mathscr{J}_{h}$ is the minimal essential ideal in $\mathbf{M}(B, R)$, where $h\left(\Lambda_{a a^{\prime}}\right)=N$, if $\Lambda_{a a^{\prime}}$ is maximal, and 0 , otherwise.

The antisymmetric radical $\mathscr{A}(\mathbf{M}(B, R))$ of a structural matrix $\operatorname{ring} \mathbf{M}(B, R)$ was introduced in [5], and is an ideal in $\mathbf{M}(B, R)$, viz the set associated with $B-C$ and $R$, where $C$ is the largest symmetric $n \times n$ Boolean matrix satisfying $C \leq B$. Then $\mathbf{M}(B, R) / \mathscr{A}(\mathbf{M}(B, R)) \cong \mathbf{M}(C, R)$.

COROLLARY 3.3. $\mathscr{A}(\mathbf{M}(B, R))$ is essential in $\mathbf{M}(B, R)$ if and only if no $\Lambda_{a a}$ is maximal, $a \in \mathbf{b}$.

Proof. Note that $\mathscr{A}(\mathbf{M}(B, R))=\mathscr{I}_{f}$, where $f\left(\Lambda_{a a^{\prime}}\right)=0$ if $a=a^{\prime}$ and $f\left(\Lambda_{a a^{\prime}}\right)=R$ if $a \neq a^{\prime}$.

Let $\Lambda_{k k}$ be maximal for some $k \in \mathbf{b}$, and let $g$ be the function mapping $\Lambda_{k k}$ onto $R$ and everything else onto 0 . Then $g$ is set-inclusion preserving and $\mathscr{I}_{f} \cap \mathscr{I}_{g}=0$, which implies that $\mathscr{I}_{f}$ is not essential.

For the converse, suppose no $\Lambda_{a a}$ is maximal, $a \in \mathbf{b}$. Then $f\left(\Lambda_{d d^{\prime}}\right)=R$ for every maximal $\Lambda_{d d^{\prime}}$, and so by Theorem $3.1, \mathscr{I}_{f}$ is essential.

EXAMPLE 3.4. If

$$
B=\left[\begin{array}{lll}
1 & 1 & 1 \\
1 & 1 & 1 \\
0 & 0 & 1
\end{array}\right]
$$

then $\sim_{B}$ induces two equivalence classes on $\{1,2,3\}$ and no $\Lambda_{a a}(=\{a\})$ is maximal, and so

$$
\mathscr{A}(\mathrm{M}(B, R))=\left[\begin{array}{lll}
0 & 0 & R \\
0 & 0 & R \\
0 & 0 & 0
\end{array}\right]
$$

is essential in $\mathbf{M}(B, R)$.

If $B$ is such that every maximal chain in the poset $\left\{z_{a}: a \in \mathbf{b}\right\}$ has precisely two elements, then $B$ is said to satisfy the pair condition. 
THEOREM 3.5. $\mathscr{A}(\mathbf{M}(B, R))$ is the minimal essential ideal in $\mathbf{M}(B, R)$ if and only if $B$ satisfies the pair condition and $R$ has no proper essential ideals.

Proof. First, if $R$ has an essential ideal $E \neq R$, then $\mathbf{M}(B-C, E) \varsubsetneqq$ $\mathbf{M}(B-C, R)=\mathscr{A}(\mathbf{M}(B, R))$. If $\mathscr{A}(\mathbf{M}(B, R))$ is essential, then by Theorem 3.1 and Corollary 3.3 the ideal $\mathbf{M}(B-C, E)$ is essential.

Secondly, suppose the pair condition does not hold and $\mathscr{A}(\mathbf{M}(B, R))$ is essential. Let $a, a^{\prime}$ and $a^{\prime \prime}$ be different elements of $\mathbf{b}$ such that $z_{a} \leq_{B} z_{a^{\prime}} \leq_{B}$ $z_{a^{\prime \prime}}, \Lambda_{a a^{\prime}}=\left\{a, a^{\prime}\right\}$ and $\Lambda_{a^{\prime} a^{\prime \prime}}=\left\{a^{\prime}, a^{\prime \prime}\right\}$. Let $f$ be as in Corollary 3.3, and let $f^{\prime}$ be the function differing from $f$ only in mapping $\Lambda_{a^{\prime} a^{\prime \prime}}$ onto 0 . It is easily checked that $f^{\prime}$ is set-inclusion preserving, and so $\mathscr{I}_{f} \subsetneq \mathscr{A}(\mathbf{M}(B, R))$. We show that $\mathscr{F}_{f}$, is essential. Let $\mathscr{I}_{g}$ be a non-zero ideal in $\mathbf{M}(B, R)$, and consider the following two possibilities: $g\left(\Lambda_{a^{\prime} a^{\prime \prime}}\right)=0$ or $g\left(\Lambda_{a^{\prime} a^{\prime \prime}}\right) \neq 0$. In the first case the construction of $f^{\prime}$ and the fact that $\mathscr{I}_{f}$ is essential show that $\mathscr{I}_{f^{\prime}} \cap \mathscr{I}_{g} \neq 0$. In the second case $g\left(\Lambda_{a a^{\prime \prime}}\right) \neq 0$, since $\Lambda_{a^{\prime} a^{\prime \prime}} \subseteq \Lambda_{a a^{\prime \prime}}$. Hence $\mathscr{I}_{f^{\prime}} \cap \mathscr{J}_{g} \neq 0$ as $f^{\prime}\left(\Lambda_{a a^{\prime \prime}}\right)=R$. Thus in both cases $\mathscr{F}_{f}$, is essential.

The converse follows directly from Theorem 3.1 and Corollary 3.3 .

The characterization of the ideals in a structural matrix ring in terms of the mentioned set-inclusion preserving functions proves immediately

PROPOSITION 3.6. $\mathcal{F}_{f}$ is small in $\mathrm{M}(B, R)$ if and only if $f\left(\Lambda_{a a}\right)$ is small in $R$ for every $a \in \mathbf{b}$.

Corollary 3 of [3] leads to the description of the Brown-McCoy radical of $\mathbf{M}(B, R)$ in terms of that of $R$, a result which can also be obtained as a special case of [5, Theorem 2.7].

$$
\text { Corollary 3.7. } \mathscr{G}(\mathbf{M}(B, R))=\mathbf{M}(B, \mathscr{G}(R))+\mathscr{A}(\mathbf{M}(B, R)) \text {. }
$$

If $B$ is symmetric, then $\mathbf{M}(B, R)$ is a direct sum of complete matrix rings, and so it is easy to see that in this case the problem of characterizing the structural matrix rings $\mathbf{M}(B, R)$ having the property that the maximal small and minimal essential ideals coincide is equivalent to the problem of characterizing the rings $R$ having this property, although $R$ is not Morita equivalent to $\mathbf{M}(B, R)$ if $B$ is not the universal $n \times n$ Boolean matrix. The main result of this section shows that the picture is different when $B$ is not symmetric.

THEOREM 3.8. If $B$ is not symmetric, then the maximal small and minimal essential ideals coincide in $\mathrm{M}(B, R)$ if and only if $B$ satisfies the pair condition and $R$ is Brown-McCoy semisimple with no proper essential ideals. 
PROOF. Note that $\mathscr{G}(\mathbf{M}(B, R))=\mathscr{I}_{g}$, where

$$
g\left(\Lambda_{a a^{\prime}}\right)= \begin{cases}\mathscr{G}(R), & \text { if } a=a^{\prime} \\ R, & \text { otherwise. }\end{cases}
$$

Since $B$ is not symmetric, $\Lambda_{d d}$ is not maximal for some $d \in \mathbf{b}$.

Suppose that $\mathscr{G}(\mathbf{M}(B, R))$ is the minimal essential ideal. Then $g=h$, with $h$ as in Corollary 3.2. Hence $\mathscr{G}(R)=0$, and so by Corollary $3.7, \mathscr{G}(\mathbf{M}(B, R))=$ $\mathscr{A}(\mathbf{M}(B, R))$. Therefore by Theorem $3.5, B$ satisfies the pair condition and $R$ has no proper essential ideals.

The converse of the assertion follows directly from Theorem 3.5 and Corollary 3.7 .

Note the similarity between the pair condition and the remark just before Theorem 2.5.

EXAMPLE 3.9. (a) Let $R$ be the quotient $\operatorname{ring} \mathbf{Z}_{m}$ of $\mathbf{Z}$, with $m=p_{1} p_{2} \cdots p_{r}$ $(r \geq 1)$ and all the $p_{i}$ prime, and let $B$ be as in Example 3.4. Then by Proposition 2.6 and Theorem 3.8 the Brown-McCoy radical

$$
\mathscr{G}\left(\mathbf{M}\left(B, \mathbf{Z}_{m}\right)\right)=\left[\begin{array}{ccc}
0 & 0 & \mathbf{Z}_{m} \\
0 & 0 & \mathbf{Z}_{m} \\
0 & 0 & 0
\end{array}\right]
$$

is the minimal essential ideal.

(b) If

$$
B=\left[\begin{array}{llll}
1 & 1 & 1 & 1 \\
1 & 1 & 1 & 1 \\
0 & 0 & 1 & 1 \\
0 & 0 & 0 & 1
\end{array}\right]
$$

then $B$ does not satisfy the pair condition, and so, for every $R$, the Brown-McCoy radical

$$
\left[\begin{array}{cccc}
\mathscr{G}(R) & \mathscr{G}(R) & R & R \\
\mathscr{G}(R) & \mathscr{G}(R) & R & R \\
0 & 0 & \mathscr{G}(R) & R \\
0 & 0 & 0 & \mathscr{G}(R)
\end{array}\right]
$$

is not the minimal essential ideal in $\mathbf{M}(B, R)$. In fact, $\mathbf{M}\left(E_{14}+E_{24}, R\right)$ is essential in $\mathbf{M}(B, R)$ and $\mathbf{M}\left(E_{14}+E_{24}, R\right) \varsubsetneqq \mathscr{G}(\mathbf{M}(B, R))$. (Here $E_{i j}$ denotes the Boolean matrix with 1 in position $(i, j)$ and 0 elsewhere.)

\section{References}

[1] V. A. Andrunakievic, 'Radicals of associative rings I', Amer. Math. Soc. Transl. (2) 52 (1966), 95-128.

[2] R. Gilmer, Multiplicative ideal theory (Marcel Dekker, New York, 1972). 
[3] N. V. Loi and R. Wiegandt, 'Small ideals and the Brown-McCoy radical', Radical Theory, (Colloq. Math. Soc. János Bolyai 38, Eger Hungary, 1982).

[4] O. F. G. Schilling, The theory of valuations (Mathematical Surveys IV, Amer. Math. Soc., 1950).

[5] L. van Wyk, 'Special radicals in structural matrix rings', Comm. Algebra 16 (1988), 421435.

Department of Mathematics

University of Stellenbosch

Stellenbosch 7600

Republic of South Africa 